\title{
面向踝关节康复的四自由度广义球面并联机构 运动学性能*
}

刘承磊 $1,2,3$ 张建军 $1,2,3$ 牛建业 ${ }^{1,2}$ 刘 腾 ${ }^{1,2}$ 戚开诚 ${ }^{1,2}$ 郭士杰 ${ }^{1,2}$

(1. 河北工业大学机械工程学院 天津 300401;

2. 河北省机器人感知与人机融合重点实验室 天津 300130;

3. 河北工业大学国家技术创新方法与实施工具工程技术研究中心 天津 300401)

\begin{abstract}
摘要: 针对现有踝关节康复机器人运动特性与人体踝关节实际运动特性存在明显差异, 导致人机相容性不理想的问题, 基于 高匹配度的 $\mathrm{U}_{1} \mathrm{U}_{2}$ 型踝关节运动拟合模型，提出一种适用于踝关节康复且结构紧凑的四自由度广义球面并联机构。基于螺旋 理论分析其运动及约束特性, 论证其与踝关节运动拟合模型运动的一致性; 分别建立机构的位置及姿态运动学模型, 证明了 该机构的位置与姿态之间运动学完全解耦; 基于雅可比矩阵极其条件数分析, 阐明该机构在纯背伸趾屈和纯内外翻运动中均 具有运动学的完全各向同性性质, 且在踝关节工作空间内具有较好的灵巧性及可操作度, 并无奇异位形等病态位置存在, 通 过数据对比验证该机构具有较好的运动学特性, 适用于踝关节康复。
\end{abstract}

关键词: 踝关节康复; 运动拟合模型; 广义球面并联机构; 运动学分析

中图分类号: TH112

\section{Kinematic Performance of 4-DOF Generalized Spherical Parallel Mechanism for Ankle Rehabilitation}

\author{
LIU Chenglei ${ }^{1,2,3}$ ZHANG Jianjun ${ }^{1,2,3}$ NIU Jianye ${ }^{1,2}$ LIU Teng ${ }^{1,2}$ \\ QI Kaicheng ${ }^{1,2}$ GUO Shijie ${ }^{1,2}$
}

(1. School of Mechanical Engineering, Hebei University of Technology, Tianjin 300401;

2. Key Laboratory of Robot Perception and Human-Machine Fusion, Hebei Province, Tianjin 300130;

3. National Engineering Research Center for Technological Innovation Method and Tool, Hebei University of

Technology, Tianjin 300401)

\begin{abstract}
In the previous rehabilitation robot research, the proposed ankle models have distinctions with the human ankle bone structure. It leads to the unsatisfactory human-machine compatibility of ankle rehabilitation robots constructed based on the fitting onto the motion of these models. A compact 4-DOF generalized spherical parallel mechanism is proposed based on the motion fitting models $U_{1} U_{2}$. In order to prove the feasibility of the mechanism, its motion and constraint characteristics are analyzed based on the screw theory, and its motion consistency with the motion fitting model is discussed. The position and orientation kinematics model of the mechanism were established separately, which proved that the kinematics between the position and orientation is completely decoupled. By analyzing its Jacobian matrix and condition number, it is clarified that the mechanism has kinematic fully-isotropic properties in single direction movement, and has excellent dexterity and manipulability in the ankle working space, and no singularity. It is verified by data comparison that the mechanism has good kinematic characteristics and is suitable for ankle rehabilitation.
\end{abstract}

Key words: ankle rehabilitation; motion fitting model; generalized spherical parallel mechanism; kinematics analysis

* 国家自然科学基金(52075145)、河北省自然科学基金重点(E2020202010)、 


\section{0 前言}

医疗康复领域的不断延伸, 正推动着踝关节康 复机器人研究展现出蓬勃态势, 促使着患者对机器 人性能及康复效果提出更高的要求。就目前而言, 针对机器人本体的构型研究已逐渐成为踝关节康 复领域的一大核心问题, 而对其本体的性能研究无 疑是该领域的重中之重。美国 Rutgers 大学 ${ }^{[1]}$ 率先 基于 Stewart 机构提出一种 6 自由度踝关节康复机 器人, 其具有自由度多、适应性强、刚度大等优点, 但由于其各支链耦合程度较强, 导致控制系统较为 复杂。故为降低机器人的构型复杂程度和控制难 度, 国内外学者相继提出了一类将动平台自由度集 中于转动自由度上的并联康复机器人, 其包括 3-SPS/S 型 ${ }^{[2]} 、 3-R S S / S$ 型 ${ }^{[3]}$ 以及 3-UPS/U 型 ${ }^{[4]}$ 康复 机器人等。但此类机构的动平台各向转动轴线与人 体踝关节实际转动轴线偏差较大, 无法支持将患者 小腿稳定放置于基座内, 导致患者很难按照专业医 生或理疗师规划的正确康复运动轨迹精确完成标 准康复训练, 影响踝关节的康复效果。为解决此类 问题, 国内外研究人员又纷纷提出了一类具有固定 远程回转中心的并联机构, 使得动平台的运动中心 始终位于踝关节运动中心附近, 大幅度提高了机器 人的人机相容性。例如: MATTEO ${ }^{[5]}$ 基于灵巧眼机 构设计的康复机器人, 边辉 ${ }^{[6]}$ 提出的 $2-R R R / U P R R$ 型康复机器人和李剑峰 ${ }^{[7-8]}$ 提出的 2-UPS/RRR 型混 联康复机器人等。此类机器人将踝关节等效为标准 球关节, 但事实上踝关节是由胫距和距下关节组成 的复合关节(广义球关节), 无论是在人体矢状面内 的背伸/跖屈运动, 或是人体冠状面内的内翻/外翻 运动, 抑或是绕人体下肢纵轴的转动, 均由胫距和 距下关节以一定关联度配合完成, 若简单将其等效 为球关节, 则仍无法避免在康复过程中产生较大的 交互力。近年来国内外学者给出了诸多极具特色的 机构本体 ${ }^{[9-17]}$ 与较为详尽的运动学分析 ${ }^{[18-24]}$, 但运 动特性与踝关节相近, 十分适用于踝关节康复的机 构本体却并不多见。

为解决此问题, 张建军等 ${ }^{[25]}$ 基于人体踝关节 骨结构提出了一系列可能的高匹配度踝关节运动 拟合模型, 如 $\mathrm{U}_{1} \mathrm{U}_{2}$ 模型, 基于此类模型提出了一 系列具有双球心的广义球面并联机构。相比较而 言此类机构作为踝关节康复机器人本体的合理程 度更高, 大幅度削弱了康复过程中的人机交互力。 但此类机构的支链耦合度仍相对较高, 控制系统
并不十分简单，且支链间易发生干涉，不利于机 器人的制造。

针对上述问题，本文拟构造一种结构紧凑且部 分解耦的踝关节康复机构。首先, 选择 $U_{1} U_{2}$ 型踝关 节运动拟合模型,在四自由度广义球面并联机构的基 础上, 提出一种结构更为紧凑的广义球面并联机构。 继而，基于螺旋理论对构造的并联机构进行约束特 性分析, 验证其运动特性与 $\mathrm{U}_{1} \mathrm{U}_{2}$ 型踝关节运动拟合 模型的一致性。最后，为阐明该机构在踝关节实际 工作空间内具有较好的解耦性、灵巧性及可操作度, 对其进行运动学分析, 从而进一步讨论该机构用于 踝关节康复的可行性。

\section{1 广义球面并联机构描述}

\section{1 踝关节运动拟合模型}

在医学上普遍定义踝关节为广义球关节，其包 括胫距关节和距下关节，胫矩关节由胫骨和距骨构 成，而距下关节则由距骨和跟骨构成，如图 1 所示。

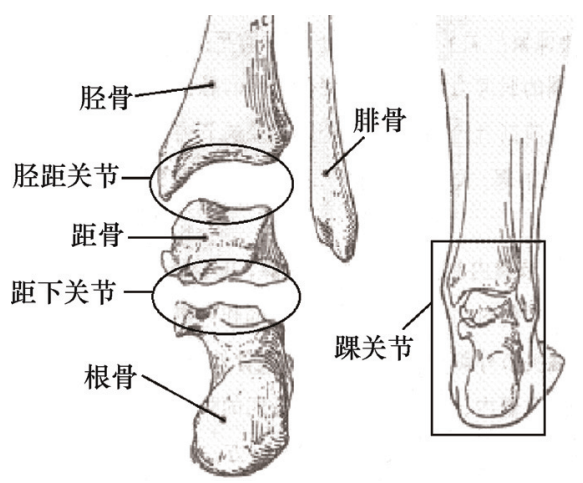

图 1 踝关节真实骨结构图

在张建军教授提出的踝关节运动拟合模型中, 将胫距关节等效为了虎克铰，而距下关节分别等效 为转动副、虎克铰和球面副, 等效胫距与距下关节 运动中心间的距离为定值且与人体距骨尺寸相匹 配, 其视为等效距骨, 如图 2 所示。

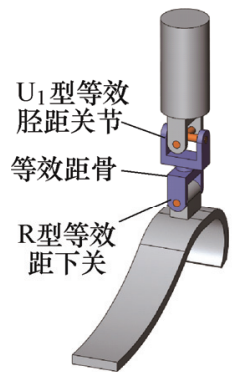

(a) $U_{1} R_{2}$

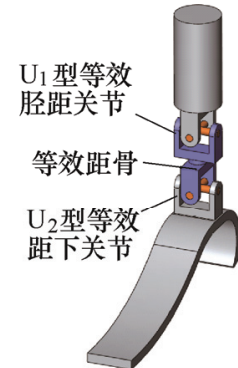

(b) $\mathrm{U}_{1} \mathrm{U}_{2}$

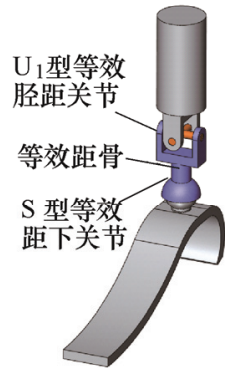

(c) $\mathrm{U}_{1} \mathrm{~S}$
图 2 踝关节等效拟合模型 


\section{2 四自由度广义球面并联机构}

由于等效距骨的尺寸较小, 如使用串联机构作 为康复机器人机构本体, 则无法在如此微小的尺 寸范围内布置足够扭矩的电机。而广义球面并联 机构具有与踝关节运动拟合模型相同的运动特 性, 故使用该机构作为踝关节康复机器人本体, 相对球面机构具有更高的拟合精度。但现有的广 义球面并联机构 ${ }^{[25]}$ 其支链相对复杂, 耦合程度较 高, 易产生支链干涉。故有必要构造一种结构紧 凑, 不易干涉, 且运动性能良好具有解耦特性的 广义球面并联机构。故以 $\mathrm{U}_{1} \mathrm{U}_{2}$ 模型为例构造一种 “结构紧凑的四自由度广义球面并联机构” 并对其 进行建模, 如图 3 所示。

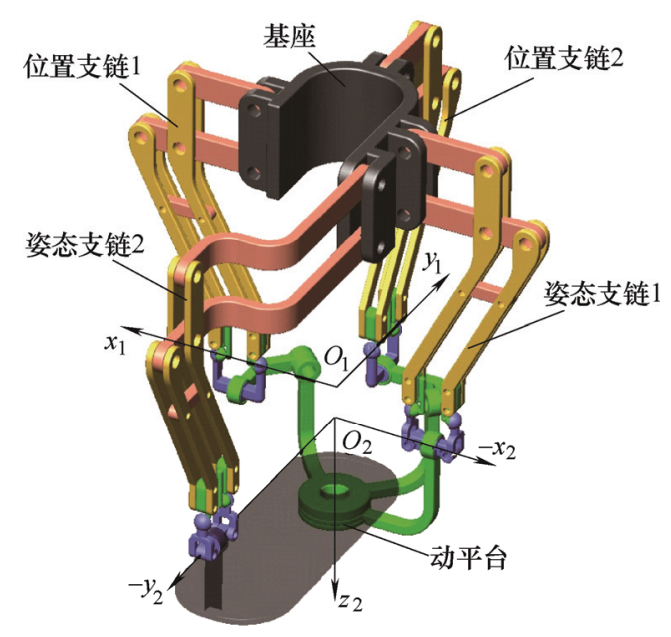

图 3 四自由度广义球面并联机构

该四自由度广义球面并联机构由一个基座、一 个动平台、两条位置支链和两条姿态支链构成。基 座与动平台内分别设有固定点 $O_{1}$ (定球心)和 $O_{2}$ (动 球心), 其分别与等效胫距关节 $\mathrm{U}_{1}$ 以及等效距下关 节 $\mathrm{U}_{2}$ 的运动中心相匹配; $O_{1}$ 与 $O_{2}$ 的连线定义为机 构的 “双心线”, 用 $O_{1} O_{2}$ 表示; 其长度定义为机构 的 “双心距”, 用 $l$ 表示。 $O_{1}$ 和 $O_{2}$ 分别与定坐标系 和动坐标系原点重合。

由于 $U_{1} U_{2}$ 型踝关节运动拟合模型的要求, 该四 自由度并联机构应具有如下特性。

(1) 由于该拟合模型的等效距骨长度固定, 故 机构双心距 $l$ 为定值且与等效距骨的长度相等。

(2) 机构具有两个移动和两个转动自由度, 即 $\mathrm{O}_{2}$ 在以 $O_{1}$ 为球心 $l$ 为半径的球面上的两自由度移 动和动平台绕 $\mathrm{O}_{2}$ 的两自由度转动, 其分别对应 $\mathrm{U}_{2}$ 关节中心的两自由度移动和 $\mathrm{U}_{2}$ 关节的两自由 度转动。

(3) 为便于运动拟合, 降低支链耦合度, 要求
所述移动与转动之间相互独立, 即实现动平台位 置、姿态间的运动学完全解耦。使两位置支链组 成的位置支链组的输入仅决定 $O_{2}$ 在球面上的位 置, 两姿态支链组成的姿态支链组的输入仅决定 动平台姿态。

\section{2 机构的约束性能分析}

机构的约束性能直接决定着机构的自由度及其 性质。此类广义球面并联机构的特点即为, 其具有 一个沿双心线 $O_{1} O_{2}$ 方向的公共约束, 故传统的 G-K 公式无法正确计算其自由度。以下基于螺旋理论进 一步分析其约束特性, 阐明其自由度与 $\mathrm{U}_{1} \mathrm{U}_{2}$ 型踝关 节运动拟合模型的一致性。

从结构上讲, 该位置和姿态支链的输入链均 引用了宗光华 ${ }^{[17]}$ 提出的 “双平行四杆 RCM 机构 (简称 RCM 机构)”。其特点为, 两球铰中心 $S_{1}$ 与 $S_{2}$ 间的距离 $l_{1}$ 为定值, 其连线始终通过远程中 心 $O_{1} ; O_{1}$ 与两个球铰中心间的距离均保持不变, 这使得轴线 $O_{1} B_{i}$ 的运动可以描述为绕 $O_{1} A_{i}$ 的转 动 $\left(O_{1} A_{i}\right.$ 为平面 $v_{i}$ 的一条法线); 其输入连杆 $a_{i}$ 始 终与轴线 $O_{1} B_{i}$ 平行, $i=1,2,3$ 或 4, 如图 4 6 所示。

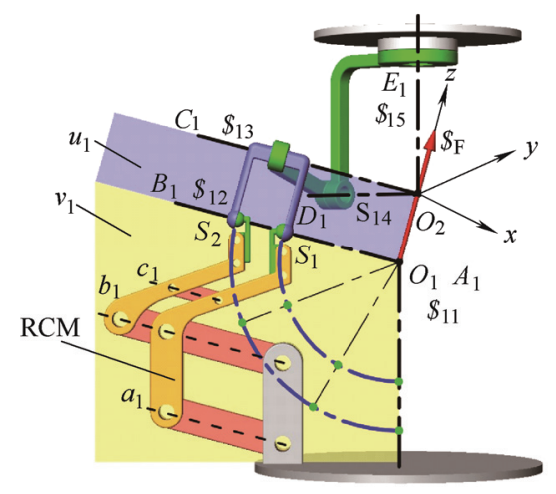

图 4 位置支链示意图

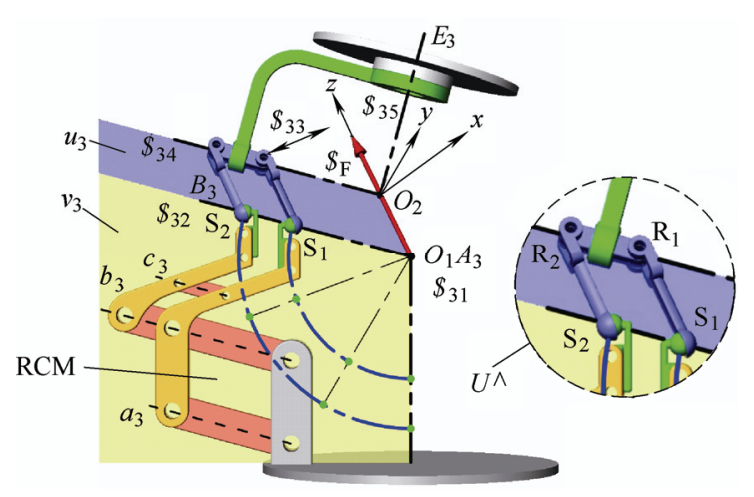

图 5 姿态支链 1 示意图 


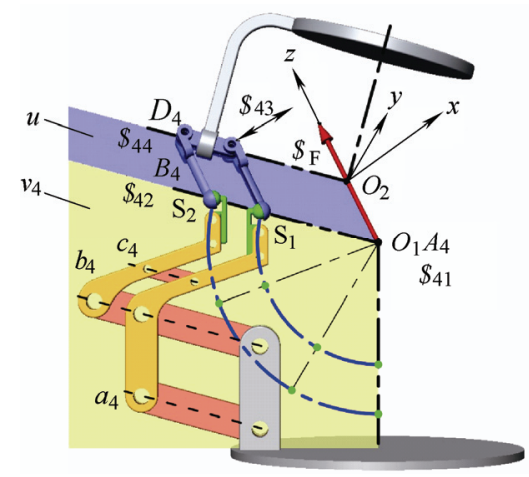

图 6 姿态支链 2 示意图

\section{1 位置支链的约束特性}

两条位置支链结构相同, 如图 4 所示, 其具有 如下特征。

(1) 法线 $O_{1} A_{1}$ 为位置支链的一条轴线。

(2) 轴线 $O_{1} A_{1}$ 始终与 $O_{1} B_{1}$ 垂直。

(3) 轴线 $O_{2} C_{1}$ 始终与 $O_{1} B_{1}$ 平行。

(4) 轴线 $O_{2} C_{1} 、 O_{2} D_{1}$ 与 $O_{2} E_{1}$ 汇交于动球心 $O_{2}$ 。

(5) 双心线 $O_{1} O_{2}$ 的长度为定值 $l$ 。

(6) 轴线 $O_{2} C_{1}$ 和 $O_{1} B_{1}$ 均与 $O_{1} O_{2}$ 垂直。

首先基于螺旋理论分析其运动螺旋, 为方便计 算将支链坐标系的原点建立在 $O_{2}$ 处, $z$ 轴与双心线 $O_{1} O_{2}$ 重合。 $\$_{11} 、 \$_{12} 、 \$_{13} 、 \$_{14}$ 与 $\$_{15}$ 分别为位置支链 的运动轴线 $O_{1} A_{1} 、 O_{1} B_{1} 、 O_{2} C_{1} 、 O_{2} D_{1}$ 与 $O_{2} E_{1}$ 在支 链坐标系内对应的运动螺旋, 其共同组成了位置支 链的运动螺旋系。 $\$_{i j}$ 表示第 $i$ 条支链的第 $j$ 个运动螺 旋。结合轴线的特殊位置关系，可计算出位置支链 的运动螺旋系为

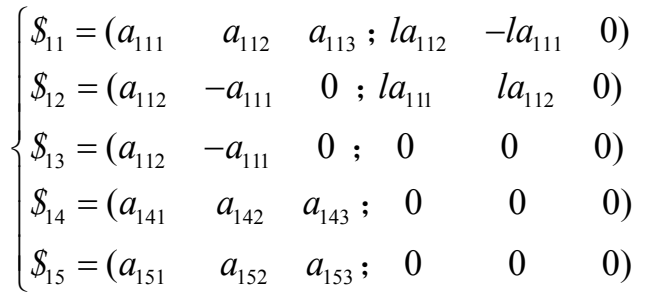

通过反螺旋计算出其约束螺旋系为

$$
\$_{11}^{r}=\left(\begin{array}{llllll}
0 & 0 & 1 ; & 0 & 0 & 0
\end{array}\right)
$$

$\$_{i j}^{r}$ 表示第 $i$ 条支链的第 $j$ 个约束螺旋。

由此分析出此类位置支链的约束螺旋系仅含有 一个沿双心线 $O_{1} O_{2}$ 方向的约束力 $\$_{\mathrm{F}}$ 。显然, 此类支 链整体在运动过程中始终位于平面 $v_{1}$ 附近, 故其具 有结构紧凑的特点。

\section{2 姿态支链 1 的约束特性}

姿态支链 1 的具体结构如图 5 所示, 其相对于 位置支链减少了一个轴线恒过 $O_{2}$ 的转动副, 增加了 一个 “等效平行四杆副 $\left(\mathrm{U}^{\wedge}\right)^{[11]}$ ”。该 $\mathrm{U}^{\wedge}$ 副中的转动 副中心 $R_{1}$ 与 $R_{2}$ 间的距离 $l_{2}$ 等于 $l_{1}, S_{1} R_{1}$ 与 $S_{2} R_{2}$ 的
长度均等于双心距 $l$ 且 $O_{2} R_{1}$ 与 $O_{1} S_{1}$ 的长度相等, 故 $S_{1} R_{1}$ 与 $S_{2} R_{2}$ 均平行于 $O_{1} O_{2}$, 显然其运动与平行四杆 副等效。由此分析出姿态支链 1 具有如下特征。

(1) 法线 $O_{1} A_{3}$ 为位置支链的一条轴线。

(2) 轴线 $O_{1} A_{3}$ 始终与轴线 $O_{1} B_{3}$ 垂直。

(3) 轴线 $O_{2} D_{3}$ 始终与 $O_{1} B_{3}$ 平行。

(4) 轴线 $O_{2} E_{3}$ 始终与 $O_{2} D_{3}$ 垂直。

(5) 双心线 $O_{1} O_{2}$ 的长度为定值 $l$ 。

(6) $\mathrm{U}^{\wedge}$ 副的平移方向始终与 $O_{1} O_{2}$ 垂直。

其支链坐标系原点仍建立在 $O_{2}$ 处, $z$ 轴沿 $O_{1} O_{2}$ 方向。其运动螺旋系相对于位置支链, 减少了一条 恒过 $O_{2}$ 的螺旋轴, 增加了一个沿 $\mathrm{U}^{\wedge}$ 副平移方向的 移动螺旋 $\$ 33$ 。综合计算出该支链的运动螺旋系为

$$
\left\{\begin{array}{l}
\$_{31}=\left(\begin{array}{llccl}
a_{311} & a_{312} & a_{313} ; l a_{312} & -l a_{311} & 0
\end{array}\right) \\
\$_{32}=\left(\begin{array}{lllcl}
a_{321} & a_{322} & a_{323} ; a_{322} & -l a_{321} & 0
\end{array}\right) \\
\$_{33}=\left(\begin{array}{ccccc}
0 & 0 & 0 ; a_{321} & a_{322} & 0
\end{array}\right) \\
\$_{34}=\left(\begin{array}{lllll}
a_{321} & a_{322} & a_{323} ; 0 & 0 & 0
\end{array}\right) \\
\$_{35}=\left(\begin{array}{lllll}
a_{351} & a_{352} & a_{353} ; 0 & 0 & 0
\end{array}\right)
\end{array}\right.
$$

通过反螺旋计算出其约束螺旋系为

$$
\$_{31}^{r}=\left(\begin{array}{llllll}
0 & 0 & 1 ; & 0 & 0 & 0
\end{array}\right)
$$

由此分析出姿态支链 1 的约束螺旋系仅含有一 个沿双心线 $O_{1} O_{2}$ 方向的约束力 $\$_{\mathrm{F}}$ 。该支链在运动过 程中整体位于平面 $v_{3}$ 附近, 故具有结构紧凑的特点。

\section{3 姿态支链 2 的约束特性}

如图 6 所示, 姿态支链 2 与 1 相比仅减少了一 条恒过 $O_{2}$ 的运动螺旋轴, 分析其运动及约束特性。

其支链坐标系原点同样建立在 $O_{2}$ 处, $z$ 轴与双 心线 $O_{1} O_{2}$ 重合。综合计算出其运动螺旋系为

$$
\left\{\begin{array}{l}
\$_{41}=\left(\begin{array}{lllll}
a_{411} & a_{412} & a_{413} ; l a_{412} & -l a_{411} & 0
\end{array}\right) \\
\$_{42}=\left(\begin{array}{lllcl}
a_{421} & a_{422} & a_{423} ; l a_{422} & -l a_{421} & 0
\end{array}\right) \\
\$_{43}=\left(\begin{array}{ccccc}
0 & 0 & 0 ; a_{421} & a_{422} & 0
\end{array}\right) \\
\$_{44}=\left(\begin{array}{lllll}
a_{421} & a_{422} & a_{423} ; 0 & 0 & 0
\end{array}\right)
\end{array}\right.
$$

通过反螺旋计算出其约束螺旋系为

$$
\left\{\begin{array}{l}
\$_{41}^{r}=\left(\begin{array}{llllll}
0 & 0 & 1 ; & 0 & 0 & 0
\end{array}\right) \\
\$_{42}^{r}=\left(\begin{array}{llllll}
0 & 0 & 0 ; & c_{421} & c_{422} & c_{423}
\end{array}\right)
\end{array}\right.
$$

其中, 参数 $c_{421} 、 c_{422}$ 以及 $c_{423}$ 需要满足

$$
\left\{\begin{array}{l}
a_{411} c_{421}+a_{412} c_{422}+a_{413} c_{423}=0 \\
a_{421} c_{421}+a_{422} c_{422}+a_{423} c_{423}=0
\end{array}\right.
$$

由此可分析出姿态支链 2 的约束螺旋系包括: 一个沿双心线 $O_{1} O_{2}$ 方向的约束力 $\$_{\mathrm{F}}$, 以及一个绕 $A_{4} O_{1} B_{4}$ 平面法线的约束力偶 $\$_{\mathrm{M}}$ 。该支链在运动过程 中整体位于平面 $v_{4}$ 附近, 故其具有结构紧凑的特点。 


\section{4 并联机构的约束特性及自由度类型}

构造机构时, 由于位置与姿态支链均具有一个 沿双心线方向的约束力且双心距 $l$ 相等, 故在动平 台和基座的约束下, 所有支链的双心线重合, 产生 一个沿 $O_{1} O_{2}$ 方向的公共约束力 $\$_{\mathrm{F}}$ (若双心距不等则 支链不能构成并联机构)。综合式(2)、(4)、(6)可计 算出并联机构的约束螺旋系, 如式(8)所示, 其具有 一个沿双心线 $O_{1} O_{2}$ 方向的约束力以及一个绕 $A_{4} O_{1} B_{4}$ 平面法线的约束力偶 $\$_{\mathrm{M}}$

$$
\left\{\begin{aligned}
& \$_{\mathrm{F}}=\left(\begin{array}{llllll}
0 & 0 & 1 ; & 0 & 0 & 0
\end{array}\right) \\
& \$_{\mathrm{M}}=\left(\begin{array}{llllll}
0 & 0 & 0 ; & c_{421} & c_{422} & c_{423}
\end{array}\right)
\end{aligned}\right.
$$

由此可知并联机构具有两个移动和两个转动自 由度。换言之, 机构的双心线 $O_{1} O_{2}$ 可绕定球心 $O_{1}$ 做两自由度转动, 动平台可绕动球心 $O_{2}$ 做两自由度 转动, 这与 $\mathrm{U}_{1} \mathrm{U}_{2}$ 型踝关节运动拟合模型具有相似的 运动特性。故从运动一致性角度讲, 该并联机构作 为踝关节康复机器人本体具有一定合理及优越性。

\section{3 机构的运动学分析}

此四自由度广义球面并联机构虽然在运动特性 上与踝关节具有较好的一致性, 但若想要以此机构 作为本体研发踝关节康复机器人, 还需对其运动性 能进行更为深入的研究。避免在康复运动中出现奇 异位形或可操作度不高等情况。

\section{1 运动学正/逆解}

该四自由度广义球面并联机构的运动学分析可 以分为两部分, 依次建立机构的位置和姿态运动学 模型。平面 $v_{1}$ 与 $v_{2}$ 相互垂直且均垂直于基座, 其分 别与踝关节矢状面与冠状面平行; 平面 $v_{3}$ 与 $v_{4}$ 相互 垂直且均垂直于基座, 其中平面 $v_{3}$ 与 $v_{1}$ 的夹角为 $180^{\circ}$, 此时平面 $v_{4}$ 与 $v_{2}$ 的夹角亦为 $180^{\circ}$ 。 $O_{1}$ 为定 坐标系原点, $z_{1}$ 轴与平面 $v_{1} 、 v_{2} 、 v_{3}$ 以及 $v_{4}$ 的交线 重合; $x_{1}$ 与 $y_{1}$ 的正半轴分别位于平面 $v_{1}$ 和 $v_{2}$ 内, 其 负半轴分别位于平面 $v_{3}$ 和 $v_{4}$ 内。 $O_{2}$ 为动坐标系原 点; $z_{2}$ 轴与 $O_{2} E_{1} 、 O_{2} E_{2}$ 和 $O_{2} E_{3}$ 轴重合并与 $O_{2} D_{4}$ 轴 垂直; $y_{2}$ 轴与 $\mathrm{O}_{2} \mathrm{D}_{4}$ 重合。

\subsection{1 位置正/逆解}

基于位置支链组构造位置运动学模型, 如图 7 所示。两位置支链组合时, 其双心线 $O_{1} O_{2}$ 重合, 结 合位置支链特征以及 RCM 机构特点, 可分析出 $O_{1} O_{2}$ 同时与输入连杆 $a_{1}$ 和 $a_{2}$ 垂直, 故 $a_{1}$ 和 $a_{2}$ 的位置参 数直接决定 $O_{1} O_{2}$ 的方向以及 $O_{2}$ 在球面上的位置; 若 $O_{2}$ 位置确定, 则定轴 $O_{2} C_{1}$ 和 $O_{2} C_{2}$ 与动轴 $O_{2} D_{1}$ 、 $O_{2} E_{1}\left(O_{2} E_{2}\right)$ 和 $O_{2} D_{2}$ 汇交于定点, 构成球面六杆闭环,
动平台仍具有三个自由度并未限制其绕 $O_{2}$ 的转动。

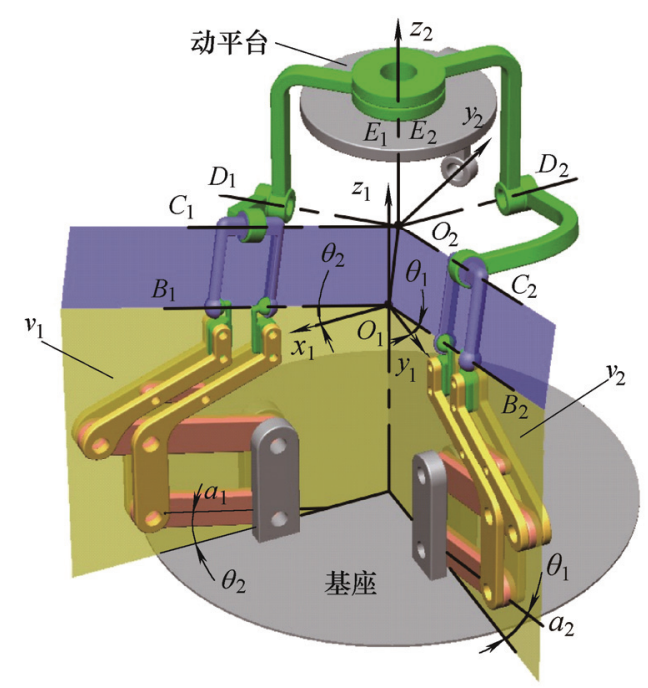

图 7 位置支链组运动学模型

$a_{1}$ 和 $a_{2}$ 在定坐标系中的向量坐标可以表示为

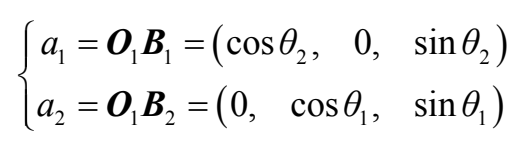

式中, $\theta_{1}$ 表示输入连杆 $a_{2}$ 与 $y_{1}$ 轴的夹角, 向上为正; $\theta_{2}$ 表示输入连杆 $a_{1}$ 与 $x_{1}$ 轴的夹角, 向上为正。

由此可轻易计算出双心线 $O_{1} O_{2}$ 在定坐标系中 的向量坐标, 继而得到 $O_{2}$ 点的坐标 $\left(m_{1}, m_{2}, m_{3}\right)$

$$
\left\{\begin{array}{l}
m_{1}=\frac{-l \cos \theta_{1} \sin \theta_{2}}{\sqrt{1-\sin ^{2} \theta_{1} \sin ^{2} \theta_{2}}} \\
m_{2}=\frac{-l \sin \theta_{1} \cos \theta_{2}}{\sqrt{1-\sin ^{2} \theta_{1} \sin ^{2} \theta_{2}}} \\
m_{3}=\frac{l \cos \theta_{1} \cos \theta_{2}}{\sqrt{1-\sin ^{2} \theta_{1} \sin ^{2} \theta_{2}}}
\end{array}\right.
$$

通过上述分析可知, $O_{2}$ 点在以 $O_{1}$ 为原点 $l$ 为半 径的球面上运动, 故使用球面坐标表示 $O_{2}$ 的位置

$$
\left(m_{1}, m_{2}, m_{3}\right)=\operatorname{Rot}\left(x, \alpha_{1}\right) \operatorname{Rot}\left(y, \beta_{1}\right)(0,0, l)^{\mathrm{T}}
$$

通过计算, 可以得到其位置解正解为

$$
\left\{\begin{array}{l}
\alpha_{1}=\theta_{1} \\
\beta_{1}=-\arctan \left(\tan \theta_{2} \cos \theta_{1}\right)
\end{array}\right.
$$

继而计算出其位置逆解为

$$
\left\{\begin{array}{l}
\theta_{1}=\alpha_{1} \\
\theta_{2}=-\arctan \left(\frac{\tan \beta_{1}}{\cos \alpha_{1}}\right)
\end{array}\right.
$$

\section{1 .2 姿态正/逆解}

基于姿态支链组构造姿态运动学模型, 如图 8 
所示。两姿态支链组合时, 其双心线 $O_{1} O_{2}$ 重合, 结 合姿态支链特征和 RCM 机构特点, 可分析出 $y_{2}$ 轴 $\left(O_{2} D_{4}\right)$ 与输入连杆 $a_{4}$ 平行, $z_{2}$ 轴同时与输入连杆 $a_{3}$ 和 $a_{4}$ 垂直, 故 $a_{3}$ 和 $a_{4}$ 的位置参数直接决定 $z_{2}$ 与 $y_{2}$ 轴的方向, 即确定了动平台姿态。

结合式(4)、(6)可计算出该姿态支链组的约束螺 旋系如式(8)所示。当输入连杆 $a_{3}$ 和 $a_{4}$ 固定导致动 平台姿态固定时, 姿态支链组的约束螺旋系增加了 三个限制动平台转动的约束螺旋, 此时经化简得到 姿态支链组的约束螺旋系为

$$
\left\{\begin{array}{l}
\$_{\mathrm{F}}=\left(\begin{array}{llllll}
0 & 0 & 1 ; & 0 & 0 & 0
\end{array}\right) \\
\$_{\mathrm{M} 1}=\left(\begin{array}{llllll}
0 & 0 & 0 ; & 1 & 0 & 0
\end{array}\right) \\
\$_{\mathrm{M} 2}=\left(\begin{array}{llllll}
0 & 0 & 0 ; & 0 & 1 & 0
\end{array}\right) \\
\$_{\mathrm{M} 3}=\left(\begin{array}{llllll}
0 & 0 & 0 ; & 0 & 0 & 1
\end{array}\right)
\end{array}\right.
$$

式(14)表示姿态支链组仅确定的动平台的姿 态, 并不影响动球心 $O_{2}$ 在球面上的位置。

$a_{3}$ 和 $a_{4}$ 在定坐标系中的向量坐标可以表示为

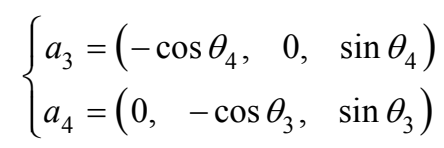

其中 $\theta_{3}$ 和 $\theta_{4}$ 分别表示输入连杆 $a_{4}$ 与 $y_{1}$ 轴负方 向的夹角以及 $a_{3}$ 与 $x_{1}$ 轴负方向的夹角, 均取向上 为正。

由此可轻易计算出 $z_{2}$ 轴在定坐标系中的向量坐 标 $\left(n_{1}, n_{2}, n_{3}\right)$

$$
\left\{\begin{array}{l}
n_{1}=\cos \theta_{3} \sin \theta_{4} \\
n_{2}=\sin \theta_{3} \cos \theta_{4} \\
n_{3}=\cos \theta_{3} \cos \theta_{4}
\end{array}\right.
$$

如图 8 所示, 基于 $\mathrm{D}-\mathrm{H}$ 法描述动平台的位姿。

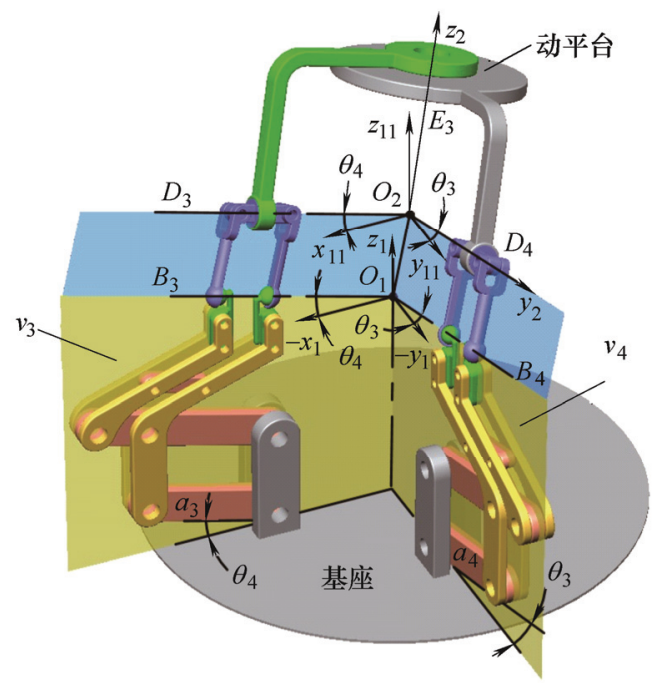

图 8 姿态支链组运动模型

(1) 首先使定坐标系沿 $O_{1} O_{2}$ 方向平移 $l$ 至 11 系,
使得 11 系的原点与 $O_{2}$ 重合。

(2) 再使 11 系围绕其自身 $x_{11}$ 轴转 $\alpha_{2}$ 角至 12 系 使得, $y_{12}$ 轴负方向与 $O_{2} D_{4}$ 轴重合, 此时 $y_{12}$ 轴同时 垂直于 $x_{12}$ 轴与 $O_{2} E_{3}$ 轴。

(3) 最后使 12 系绕其自身 $y_{12}$ 轴旋转 $\beta_{2}$ 角至动 坐标系( 2 系), 使动坐标系的 $z_{2}$ 轴与 $O_{2} E_{3}$ 轴重合。

由此可以得到此类机构的位姿矩阵

$$
\boldsymbol{T}=\operatorname{Tran}\left(m_{1}, m_{2}, m_{3}\right) \operatorname{Rot}\left(x, \alpha_{2}\right) \operatorname{Rot}\left(y, \beta_{2}\right)
$$

式中, $\left(m_{1}, m_{2}, m_{3}\right)$ 如式(11)所示。

通过计算, 得到其姿态正解为

$$
\left\{\begin{array}{l}
\alpha_{2}=-\theta_{3} \\
\beta_{2}=\arctan \left(\tan \theta_{4} \cos \theta_{3}\right)
\end{array}\right.
$$

进而计算出其姿态逆解为

$$
\left\{\begin{array}{l}
\theta_{3}=-\alpha_{2} \\
\theta_{4}=\arctan \left(\frac{\tan \beta_{2}}{\cos \alpha_{2}}\right)
\end{array}\right.
$$

\section{2 解偶特性分析}

综上，式(12)、(18)组成该机构运动学正解; 式(13)、(19)组成运动学逆解。显然此四自由度广义 球面并联机构实现了位置和姿态间的完全解耦。为 进一步研究并联机构的驱动与踝关节运动拟合模型 运动的解耦关系, 建立四自由度广义球面并联机构 与 $\mathrm{U}_{1} \mathrm{U}_{2}$ 型踝关节运动拟合模型的运动系统模型, 如 图 9 所示。在运动过程中位置支链 1、位置支链 2、 姿态支链 1 与姿态支链 2 整体分别位于冠状面 $v_{1}$ 、 矢状面 $v_{2}$ 、冠状面 $v_{3}$ 与矢状面 $v_{4}$ 附近, 故此机构不 易产生干涉现象。

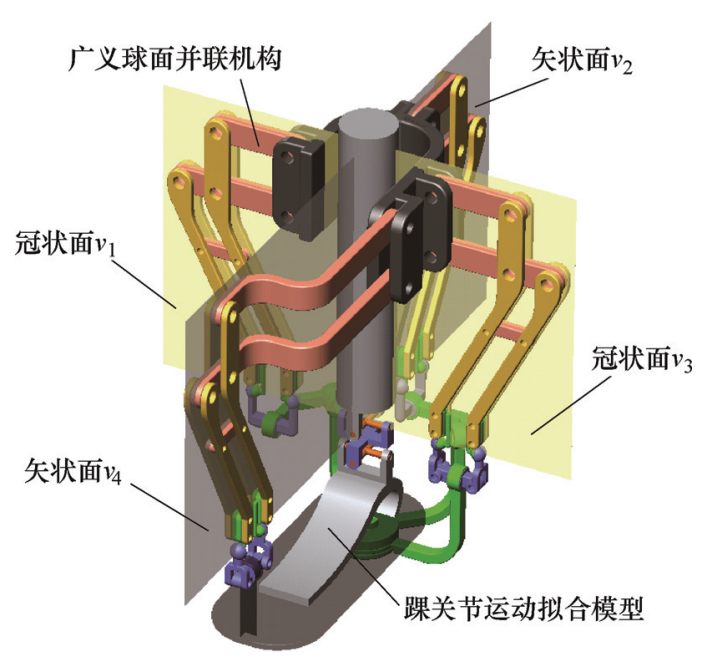

图 9 广义球面并联机构与踝关节运动拟合模型系统

显然系统动平台的位姿参数中, $\alpha_{1}$ 主要描述等 
效距骨的背伸趾屈运动, $\alpha_{2}$ 主要描述脚掌的背伸趾 屈运动, 故将其共同定义为机构的 “背伸趾屈角”, 如图 10a 所示; $\beta_{1}$ 主要描述等效距骨的内外翻运动, $\beta_{2}$ 主要描述脚掌的内外翻运动, 故将其定义为机构 的 “内外翻角”, 如图 $10 \mathrm{~b}$ 所示。

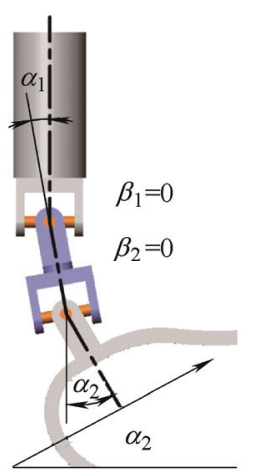

(a) 纯背伸趾屈

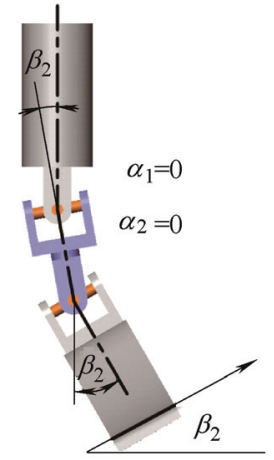

(b) 纯内翻外翻屈
图 10 拟合模型运动参数示意图

将系统的 “纯背伸趾屈” 和 “纯内外翻” 运动 统一定义为系统的 “单一运动”。所述的 “纯背伸趾 屈” 指踝关节仅在矢状面内的运动, 即满足 $\beta_{1}=0$ 且 $\beta_{2}=0$, 如图 10a 所示, 结合式(13)、(19)可分析出此 时输入转角 $\theta_{2}$ 与 $\theta_{4}$ 均为零, 并且 $\theta_{1}=\alpha_{1} 、 \theta_{3}=-\alpha_{2}$, 机构处于完全解耦状态; “纯内翻外翻” 指踝关节仅 在冠状面内的运动, 即满足 $\alpha_{1}=0$ 且 $\alpha_{2}=0$, 如图 $10 \mathrm{~b}$ 所示, 结合式(13)、(19)可分析出此时输入转角 $\theta_{1}$ 与 $\theta_{3}$ 均为零, 并且 $\theta_{2}=-\beta_{1} 、 \theta_{4}=\beta_{2}$, 机构同样处于 完全解耦状态。

综上, 此并联机构和踝关节运动拟合模型组成 的系统具有单一运动下的完全解耦特性, 该解偶特 性将大幅度降低康复机器人控制系统的复杂程度, 具有一定的实际意义。

\section{3 速度雅可比矩阵及灵巧性分析}

通过对式(13)、(19)进行微分运算得到并联机构 输入和输出的速度关系式为

$$
\left\{\begin{array}{l}
\dot{\theta}_{1}=\dot{\alpha}_{1} \\
\dot{\theta}_{2}=\frac{\dot{\alpha}_{1} \sin \beta_{1} \cos \beta_{1} \sin \alpha_{1}+\dot{\beta}_{1} \cos \alpha_{1}}{\sin ^{2} \alpha_{1} \cos ^{2} \beta_{1}-1} \\
\dot{\theta}_{3}=-\dot{\alpha}_{2} \\
\dot{\theta}_{4}=-\frac{\dot{\alpha}_{2} \sin \beta_{2} \cos \beta_{2} \sin \alpha_{2}+\dot{\beta}_{2} \cos \alpha_{2}}{\sin ^{2} \alpha_{2} \cos ^{2} \beta_{2}-1}
\end{array}\right.
$$

并联机构的速度雅可比矩阵 $\boldsymbol{J}$ 表示动平台输出 速度和驱动速度的线性变换关系, 其满足

$$
\left(\begin{array}{llll}
\dot{\theta}_{1} & \dot{\theta}_{2} & \dot{\theta}_{3} & \dot{\theta}_{4}
\end{array}\right)^{\mathrm{T}}=\boldsymbol{J}\left(\begin{array}{llll}
\dot{\alpha}_{1} & \dot{\beta}_{1} & \dot{\alpha}_{2} & \dot{\beta}_{2}
\end{array}\right)^{\mathrm{T}}
$$

基于式(20)、(21)即可计算出该雅可比矩阵 $J$ 如
式(22)所示

$$
\boldsymbol{J}=\left[\begin{array}{cccc}
1 & 0 & 0 & 0 \\
A_{21} & A_{22} & 0 & 0 \\
0 & 0 & -1 & 0 \\
0 & 0 & A_{43} & A_{44}
\end{array}\right]
$$

式(22)中的参数 $A_{21} 、 A_{22} 、 A_{43}$ 和 $A_{44}$ 与位姿参 数 $\alpha_{1} 、 \beta_{1} 、 \alpha_{2}$ 和 $\beta_{2}$ 之间的关系依次表示为

$$
\left\{\begin{array}{l}
A_{21}=\frac{\sin \beta_{1} \cos \beta_{1} \sin \alpha_{1}}{\sin ^{2} \alpha_{1} \cos ^{2} \beta_{1}-1} \\
A_{22}=\frac{\cos \alpha_{1}}{\sin ^{2} \alpha_{1} \cos ^{2} \beta_{1}-1} \\
A_{43}=-\frac{\sin \beta_{2} \cos \beta_{2} \sin \alpha_{2}}{\sin ^{2} \alpha_{2} \cos ^{2} \beta_{2}-1} \\
A_{44}=-\frac{\cos \alpha_{2}}{\sin ^{2} \alpha_{2} \cos ^{2} \beta_{2}-1}
\end{array}\right.
$$

继而计算出该速度雅可比矩阵的条件数

$$
\left\{\begin{array}{l}
\lambda_{1}=1 \\
\lambda_{2}=\frac{\cos \alpha_{1}}{\sin ^{2} \alpha_{1} \cos ^{2} \beta_{1}-1} \\
\lambda_{3}=-1 \\
\lambda_{4}=-\frac{\cos \alpha_{2}}{\sin ^{2} \alpha_{2} \cos ^{2} \beta_{2}-1}
\end{array}\right.
$$

如式(24)所示, $\lambda_{1}=1$ 且 $\lambda_{3}=-1$ 恒成立说明此机 构在纯背伸趾屈运动中具有极好的灵巧性, 其处于 运动学完全各向同性。 $\lambda_{2}$ 仅与位置参数 $\alpha_{1}$ 和 $\beta_{1}$ 相 关, $\lambda_{4}$ 仅与姿态参数 $\alpha_{2}$ 和 $\beta_{2}$ 相关; 当 $\theta_{1}=\alpha_{1}=0$ 时, 有 $\lambda_{2}=1$; 当 $\theta_{3}=\alpha_{2}=0$ 时, 有 $\lambda_{4}=-1$, 这说明机构在 纯内外翻状态时, 同样具有完全各向同性。故该四 自由度广义球面并联机构具有单一运动下的完全各 向同性，其灵巧性相对良好。

\section{4 机构的可操作度分析}

机构的可操作度 $k$ 用于描述机构在各个方向上 的运动综合性和整体灵活性, 可使用雅可比矩阵的 行列式值表示(其为无量纲数)。可操作度为 1 时机 构具有最佳的可操作性能; 若可操作度 $k$ 趋近于零 或无穷, 该雅可比矩阵属于病态矩阵, 机构的输入 和输出运动的映射关系出现失真, 机构接近于奇异 位形。为避免这一现象, 需要对机构的可操作度进 行进一步分析。

经计算, 机构的可操作度为

$$
k=\sqrt{\operatorname{det}\left(\boldsymbol{J} \boldsymbol{J}^{\mathrm{T}}\right)}=\left|\lambda_{2}\right|\left|\lambda_{4}\right|
$$

由于 $\lambda_{1}$ 仅与位置参数 $\alpha_{1}$ 和 $\beta_{1}$ 相关, $\lambda_{2}$ 仅与姿 态参数 $\alpha_{2}$ 和 $\beta_{2}$ 相关, 令 $k_{1}=\left|\lambda_{2}\right| 、 k_{2}=\left|\lambda_{4}\right|$, 即有 $k=k_{1} k_{2}$ 。 
其中 $k_{1}$ 为该并联机构的位置可操作度, 其分布情况 可以描述踝关节等效距骨的整体灵活性; $k_{2}$ 为该机 构的姿态可操作度, 其分布规律可描述根骨及脚掌 的整体灵活度。

$$
\left\{\begin{array}{l}
k_{1}=\left|\frac{\cos \alpha_{1}}{1-\sin ^{2} \alpha_{1} \cos ^{2} \beta_{1}}\right| \\
k_{2}=\left|\frac{\cos \alpha_{2}}{1-\sin ^{2} \alpha_{2} \cos ^{2} \beta_{2}}\right|
\end{array}\right.
$$

分别研究 $k_{1}$ 和 $k_{2}$ 在半球域内的分布情况, 如 图 11、12 所示。半球域则表示 $\alpha_{1} 、 \beta_{1} 、 \alpha_{2}$ 和 $\beta_{2}$ 均 限制在- $90^{\circ} \sim 90^{\circ}$ 范围内时的工作空间。

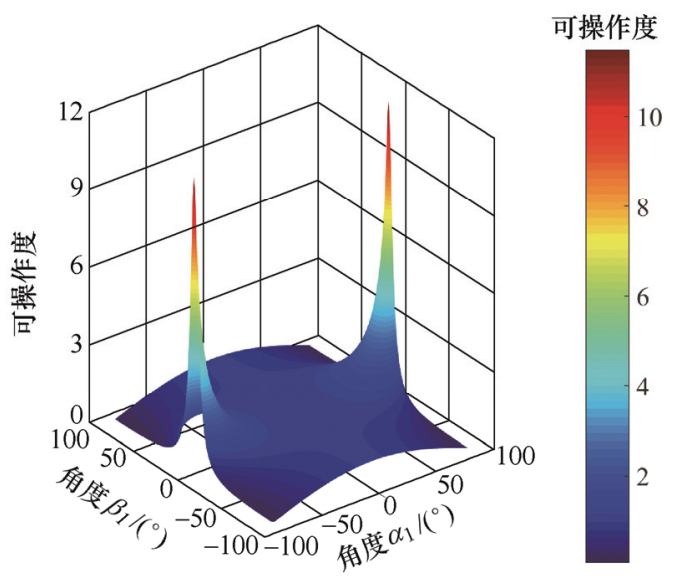

图 11 半球域内的 $k_{1}$ 值分布图

从图 11 中可看出 $\left(\alpha_{1}, \beta_{1}\right)$ 在点 $\left(-90^{\circ},-90^{\circ}\right) 、\left(-90^{\circ}\right.$, $\left.90^{\circ}\right) 、\left(90^{\circ},-90^{\circ}\right)$ 和 $\left(90^{\circ}, 90^{\circ}\right)$ 附近的位置可操作度 $k_{1}$ 趋近于零, 而在 $\left(-90^{\circ}, 0\right)$ 和 $\left(90^{\circ}, 0\right)$ 附近的 $k_{1}$ 值趋近 于无穷。故此六个位置即为该并联机构的位置奇异 位形。

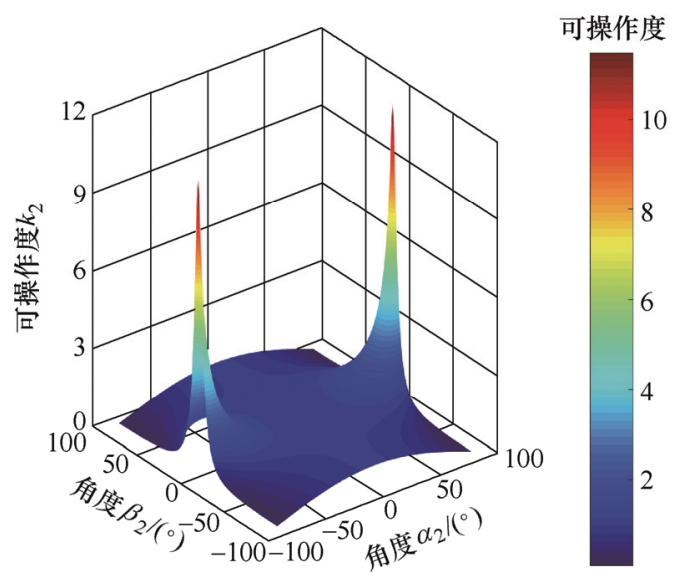

图 12 半球域内的 $k_{2}$ 值分布图

从图 12 中可看出 $\left(\alpha_{2}, \beta_{2}\right)$ 在点 $\left(-90^{\circ},-90^{\circ}\right) 、\left(-90^{\circ}\right.$, $\left.90^{\circ}\right) 、\left(90^{\circ},-90^{\circ}\right)$ 和 $\left(90^{\circ}, 90^{\circ}\right)$ 附近的姿态可操作度 $k_{2}$
趋近于零, 而在 $\left(-90^{\circ}, 0\right)$ 和 $\left(90^{\circ}, 0\right)$ 附近的 $k_{2}$ 值趋近 于无穷。故此六个位置为该并联机构的姿态奇异 位形。

显然, 该机构所有的奇异位形均分布在半球域 的边缘处，而实际上，人体踝关节的实际运动空间 普遍集中在半球域的中心附近。表 1 给出了在行走 步态中踝关节距骨的背伸趾屈和内外翻的极限角 度以及根骨的背伸趾屈和内外翻的极限角度 ${ }^{[26-27]}$, 其分别对应 $\alpha_{1}$ 与 $\beta_{1}$ 的运动范围及 $\alpha_{2}$ 与 $\beta_{2}$ 的运动 范围。

\section{表 1 踝关节运动范围}

\begin{tabular}{ccc}
\hline 运动类型 & 距骨范围 $/\left(^{\circ}\right)$ & 根骨范围 $/\left(^{\circ}\right)$ \\
\hline 背伸 & $9.81 \sim 12.57$ & $20.3 \sim 29.8$ \\
趾屈 & $4.53 \sim 13.43$ & $37.6 \sim 45.8$ \\
内翻 & $2.54 \sim 5.10$ & $25.0 \sim 30.0$ \\
外翻 & $1.03 \sim 1.91$ & $10.0 \sim 17.0$ \\
\hline
\end{tabular}

由表 1 可以得到位姿参数的边界条件如下

$$
\begin{aligned}
& \alpha_{1} \in\left(-12.57^{\circ}, 13.43^{\circ}\right) \\
& \beta_{1} \in\left(-5.10^{\circ}, 1.91^{\circ}\right) \\
& \alpha_{2} \in\left(-29.80^{\circ}, 45.80^{\circ}\right) \\
& \beta_{2} \in\left(-30.0^{\circ}, 17.0^{\circ}\right)
\end{aligned}
$$

在上述运动范围内进一步研究 $k_{1}$ 和 $k_{2}$ 在工作空 间内的分布情况，如图 13、14 所示。

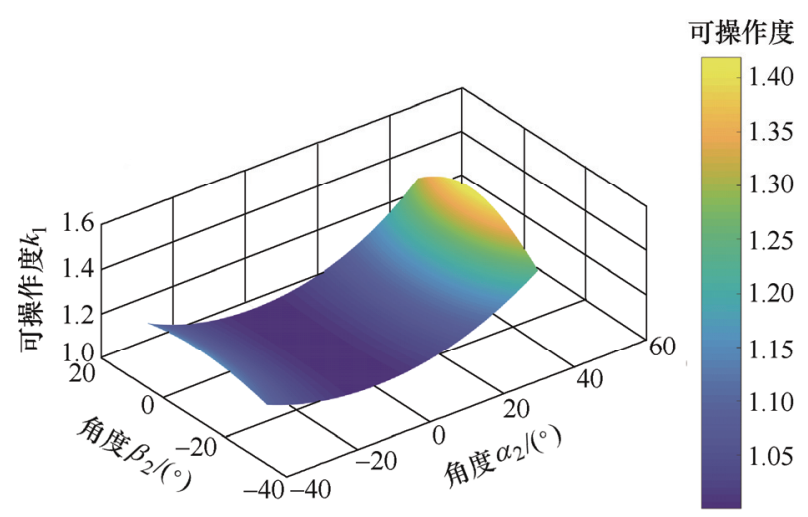

图 13 距骨工作空间内的 $k_{1}$ 值分布

图 13 展示了 $k_{1}$ 在距骨运动空间内的分布情况, 其值在 $1 \sim 1.45$ 之间, 且初始平衡位置时, 接近于 1 。

图 14 展示了 $k_{2}$ 在踝关节跟骨运动空间内的分 布情况, 显示其值在 $1 \sim 1.03$ 之间, 且越接近中心(起 始)位置其值越接近于 1 。根据 $k=k_{1} k_{2}$ 计算出, 该机 构的可操作度 $k$ 值分布在 $1 \sim 1.5$ 之间, 并且在工作 空间内变化平稳连续, 故不存在奇异位形。 


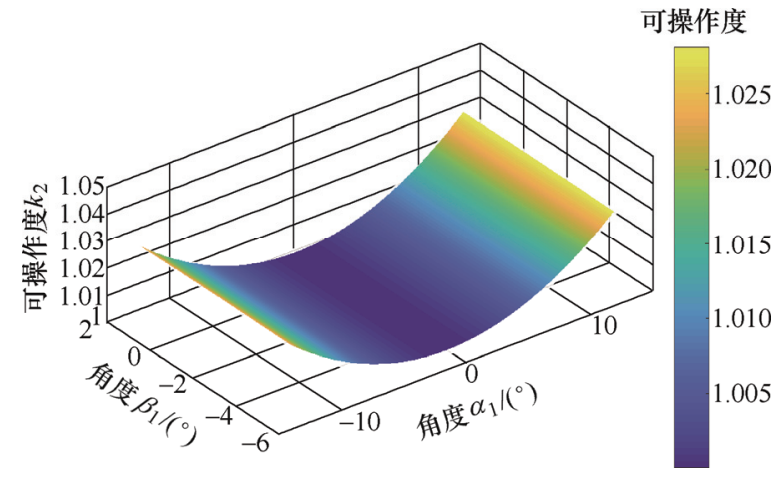

图 14 跟骨工作空间内的 $k_{2}$ 值分布

以目前较为先进的 2-UPS/RRR型踝关节康复 机器人为例, 其在踝关节运动空间内的可操作度基 本在 $1.5 \sim 4.5$ 之间 ${ }^{[8]}$ 。显然, 与其相比, 四自由度 广义球面机构的可操作度更接近于 1 , 其具有更为 优异的运动综合性和整体灵活性, 故优越性较为 突出。

\section{4 结论}

(1) 在 $U_{1} U_{2}$ 型踝关节运动拟合模型的基础上, 提出了一种结构紧凑的四自由度广义球面并联机 构, 并建立了以其为机构本体的踝关节康复机器人 模型。

（2）基于螺旋理论分析了该机构运动及约束特 性, 阐明了该机构的自由度及其类型, 证明其运动 特性与 $\mathrm{U}_{1} \mathrm{U}_{2}$ 型踝关节运动拟合模型相同。

(3) 分别建立了位置和姿态运动学模型, 基于 D-H 法分析了位置及姿态的正/逆运动学。基于雅可 比矩阵及其条件数, 阐明在纯背伸趾屈和纯内外翻 运动中, 机构的运动学处于完全解耦, 具有完全各 向同性、灵巧性极佳; 分析了机构的位置可操作度 $k_{1}$ 和姿态可操作度 $k_{2}$ 在半球域内的分布规律, 证明 了机构的奇异位形均分布在半球域的边缘。

（4）根据人体踝关节距骨及根骨的实际运动范 围, 给出了可操作度在踝关节实际工作空间内的分 布规律, 计算出其值分布在 $1 \sim 1.5$ 之间, 说明该机 构在实际工作空间内具有较好的运动学特性。证明 了此机构用于踝关节康复的可行性, 为此类康复机 器人的本体研发打下良好基础。

\section{参 考 文 献}

[1] BOIAN R F, BOUZIT M, BURDEA G C, et al. Dual Stewart platform mobility simulator[J]. Conf. Proc. IEEE Eng. Med. Biol. Soc., 2004, 2(7): 4848-4851.

[2] DAI J S, ZHAO T S, NESTER C. Sprained ankle physiotherapy based mechanism synthesis and stiffness analysis of a robotic rehabilitation device[J]. Autonomous Robots, 2004, 16(2): 207-218.

[3] 于海波, 赵铁石, 李仕华, 等. 空间 3-SPS/S 对顶双雉 机构的运动学分析[J]. 机械设计, 2017, 24(2): 11-14. YU Haibo, ZHAO Tieshi, LI Shihua, et al. Kinematics analysis on the spatial 3-SPS/S opposing vertexes double pyramid mechanism[J]. Journal of Machine Design, 2017, 24(2): 11-14.

[4] SAGLiA J A, TSAGARAKIS N G, DAI J S, et al. A high-performance redundantly actuated parallel mechanism for ankle rehabilitation[J]. International Journal of Robotics Research, 2009, 28(9): 1216-1227.

[5] MALOSIO M, NEGRI S P, PEDROCCHI N, et al. A spherical parallel three degrees-of-freedom robot for ankle-foot neuro-rehabilitation[C]// Annual International Conference of the IEEE Engineering in Medicine and Biology Society. Milan, Italy: IEEE Engineering in Medicine and Biology Society, 2012: 3356-3359.

[6] 边辉, 刘艳辉, 梁志成. 并联 2-RRR/UPRR 踝关节康复 机器人机构及其运动学 $[\mathrm{J}]$. 机器人, 2010, 32(1): 6-12. BIAN Hui, LIU Yanhui, LIANG Zhicheng. A novel 2-RRR/UPRR robot mechanism for ankle rehabilitation and its kinematics[J]. Robot, 2010，32(1): 6-12.

[7] 李剑锋, 张凯, 张雷雨. 并联踝康复机器人的设计与运 动性能评价[J]. 机械工程学报，2019，55(9)：29-39.

LI Jianfeng, ZHANG Kai, ZHANG Leiyu. Design and kinematic performance evaluation of parallel ankle rehabilitation robot[J]. Journal of Mechanical Engineering, 2019, 55(9): 29-39.

[8] 李剑锋, 李世才, 陶春静. 并联 2-UPS/RRR 踝关节康 复机构及运动性能分析 $[\mathrm{J}]$. 机器人, 2016, 38(2): 144-153.

LI Jianfeng, LI Shicai, TAO Chunjing. Parallel 2-UPS/RRR ankle rehabilitation mechanism and kinematic performance analysis[J]. Robot, 2016, 38(2): 144-153.

[9] HUANG Z, LI Q C. General methodology for type synthesis of lower-mobility symmetrical parallel manipulators and several novel manipulators[J]. International Journal of Robotics Research, 2002, 21(2): 131-146.

[10] YANG S F, SUN T, HUANG T. Type synthesis of parallel mechanisms having 3T1R motion with variable rotational axis[J]. Mechanism and Machine Theory, 2017, 109: 220-230.

[11] GAO F, LI W M, ZHAO X C, et al. New kinematic structures for 2-, 3-, 4-, and 5-dof parallel manipulator designs[J]. Mechanism and Machine Theory, 2002, 37(11): 1395-1411. 
[12] KONG X W, GOSSELIN C M. Type synthesis of 3T1R 4-DOF parallel manipulators based on screw theory[J]. IEEE Transactions on Robotics and Automation, 2004, 20(2): 181-190.

[13] FANG Y F, TSAI L W. Structure synthesis of a class of 4-degree of freedom and 5-degree of freedom parallel manipulators with identical limb structures[J]. International Journal of Robotics Research，2002，21(9): 799-810

[14] LI Q C, HERVE J M. Parallel mechanisms with bifurcation of schoenflies motion[J]. IEEE Transactions on Robotics, 2009, 25(1): 158-164.

[15] XIE F G, LI T, LIU X J. Type synthesis of 4-DOF parallel kinematic mechanisms based on Grassmann line geometry and atlas method[J]. Chinese Journal of Mechanical Engineering, 2013, 26(6): 1073-1081.

[16] WU K, YU J J, ZONG G H, et al. A family of rotational parallel manipulators with equal-diameter spherical pure rotation[J]. Journal of Mechanisms and Robotics, 2013, 6(1): 011008.

[17] 宗光华, 裴旭, 于靖军, 等. 双平行四杆型远程运动中 心机构的设计 [J]. 机械工程学报, 2007, 43(12): 103-108.

ZONG Guanghua, PEI Xu, YU Jingjun, et al. Design of double parallelogram remote-center-of-motion mechanisms[J]. Journal of Mechanical Engineering, 2007, 43(12): 103-108.

[18] 叶伟, 杨臻, 李秦川. 一种远中心并联机构运动学与性 能分析[J]. 机械工程学报, 2019，55(5): 65-73.

YE Wei, YANG Zhen, LI Qinchuan. Kinematics and performance analysis of a parallel manipulator with remote center of motion[J]. Journal of Mechanical Engineering, 2019, 55(5): 65-73.

[19] 李秦川, 孙晓东, 陈巧红, 等. 2-PRS-PRRU 并联机构 运动学与奇异分析 $[\mathrm{J}]$. 机械工程学报, 2011，47(3): 21-27.

LI Qinchan, SUN Xiaodong, CHEN Qiaohong, et al. Kinematics and singularity analysis of 2-PRS-PRRU parallel mechanism[J]. Journal of Mechanical Engineering, 2011, 47(3): 21-27.

[20] 贺磊盈, 涂叶凯, 叶伟, 等. 一种可整周回转的新型 3T1R 并联机构运动学分析[J]. 机械工程学报, 2018, 54(11): 151-160.

HE Leiying, TU Yekai, YE Wei, et al. Kinematics analysis of a novel 3T1R parallel manipulator with full rotational capability[J]. Journal of Mechanical Engineering, 2018, 54(11): 151-160.
[21] 吴存存, 杨桂林, 陈庆盈, 等. 四自由度 $2 \mathrm{PPPaR}$ 并联 机构运动学及性能分析 [J]. 机械工程学报, 2018, 54(3): 36-45.

WU Cuncun, YANG Guilin, CHEN Qingying, et al. Kinematic and performance analysis of a 4-DOF $2 \mathrm{PPPaR}$ parallel manipulator[J]. Journal of Mechanical Engineering, 2018, 54(3): 36-45.

[22] 沈惠平, 许正骁, 许可, 等. 低耦合度且部分解耦的 3T1R 并联机构设计与分析[J]. 农业机械学报, 2019,

50(2): 373-383.

SHEN Huiping, XU Zhengxiao, XU Ke, et al. Design and analysis for partially decoupled 3T1R parallel mechanism with low coupling degree $[\mathrm{J}]$. Journal of Mechanical Engineering, 2019, 50(2): 373-383.

[23] DONG X, YU J J, CHEN B, et al. Geometric approach for kinematic analysis of a class of 2-DOF rotational parallel manipulators[J]. Chinese Journal of Mechanical Engineering, 2012, 25(2): 241-247.

[24] 陈斌，于靖军，宗光华，等. 一类 2 自由度 $n-4 \mathrm{R}$ 并联 指向机构的运动学分析 [J]. 机械工程学报, 2014, 50(15): 19-27.

CHEN Bin, YU Jinjun, ZONG Guanghua, et al. Kinematic analysis of a class of 2-DOF $n-4 \mathrm{R}$ parallel pointing mechanism[J]. Journal of Mechanical Engineering, 2014, 50(15): 19-27.

[25] 刘承否, 张建军, 戚开诚, 等. 面向踝部康复的广义球 面并联机构型综合 $[\mathrm{J}]$. 机械工程学报，2020，56(19): 79-91.

LIU Chenglei, ZHANG Jianjun, QI Kaicheng, et al. Synthesis of generalized spherical parallel manipulations for ankle rehabilitation[J]. Journal of Mechanical Engineering, 2020, 56(19): 79-91.

[26] TSOI Y H, XIE S Q. Design and control of a parallel robot for ankle rehabilitation[J]. International Journal of Intelligent Systems Technologies and Applications, 2010, $8(1 / 2 / 3 / 4):$ : 100-113.

[27] LEE Y, CHEN K, REN Y, et al. Robot-guided ankle sensorimotor rehabilitation of patients with multiple sclerosis[J]. Multiple Sclerosis and Related Disorders, 2017, 11(65): 1-27.

作者简介: 刘承否, 男, 1995 年出生, 博士研究生。主要研究方向为并 联机器人。

E-mail: 15620196305@163.com

张建军(通信作者), 男, 1971 年出生, 博士, 教授, 博士研究生导师。 主要研究方向为机器人机构学、并联机器人、外骨骼机器人。

E-mail: zhjjun@hebut.edu.cn 